\title{
Polimorfismos del gen ApoE en individuos con síndrome de Down y sus progenitores en una población colombiana
}

\author{
Lucero Rengifo, Duverney Gaviria, Herman Serrano \\ Centro de Biología Molecular y Biotecnología, Universidad Tecnológica de Pereira, Pereira, Colombia
}

Introducción. Los polimorfismos en el gen $A p o E$ se han examinado en el síndrome de Down debido a la relación existente de la isoforma E4 con la demencia de tipo Alzheimer que aparece en los individuos con síndrome de Down.

Objetivos. Determinar los polimorfismos en el gen ApoE en individuos con síndrome de Down y sus progenitores, y buscar su asociación.

Materiales y métodos. Mediante PCR-RFLP, se analizaron los polimorfismos del gen ApoE en 134 individuos jóvenes con síndrome de Down, 87 madres y 54 padres del eje cafetero, y se compararon con una población control de 525 individuos sanos.

Resultados. El alelo APOEع3 y el genotipo $\varepsilon 3 / \varepsilon 3$ fueron los más frecuentes en todas las poblaciones. La frecuencia alélica de APOEع2 es muy baja y $\varepsilon 2 / \varepsilon 2$ está ausente en las poblaciones con síndrome de Down y sus progenitores. El alelo APOE\&4 fue más frecuente en individuos con síndrome de Down que en el resto de poblaciones analizadas. Al comparar las frecuencias alélicas y genotípicas entre las poblaciones con síndrome de Down y los progenitores con la población control, mediante la $\chi^{2}$ de Pearson y los odds ratios por la prueba exacta de Fisher, no se encontraron diferencias estadísticamente significativas.

Conclusiones. No se encontró asociación entre los polimorfismos del gen ApoE y el síndrome de Down. Es posible que el tamaño de la muestra o las influencias étnicas hubieran afectado estos resultados. Es necesario hacer otros estudios en poblaciones colombianas y evaluar la asociación con otros genes que se encuentran relacionados con la enfermedad de Alzheimer.

Palabras clave: apolipoproteínas E, síndrome de Down, enfermedad de Alzheimer, alelos, genotipo, Colombia.

\section{APOE gene polymorphisms associated with Down syndrome in Colombian populations}

Introduction. Gene APOE\&4 allele polymorphisms have been examined in Down syndrome because of the relationship between (a) the E4 isoform and (b) the type of Alzheimer's dementia that appears in individuals with Down syndrome. This isoform is considered a risk factor for Alzheimer's disease development and has been associated with early death in Down syndrome.

Objectives. The polymorphisms in the APOE gene were characterized for Down syndrome individuals and their parents, in order to detect associations between the APOE polymorphisms and Down syndrome.

Materials and methods. APOE gene polymorphisms were detected by RFLP-PCR and analyzed in 134 young individuals with Down syndrome, 87 mothers and 54 fathers, residents of the departments of Quindío and Risaralda, Colombia. The controls were 525 healthy individuals.

Results. The APOE\&3 allele and $\varepsilon 3 / \varepsilon 3$ genotype were most frequent in all the populations ( $83-90 \%$ and $70-78 \%$ ). The allelic frequency of APOEع2 was very low and $\varepsilon 2 / \varepsilon 2(3-7 \%)$ was absent in Down syndrome and their parents. The allele APOE\&4 was more frequent $(11 \%$ vs. $9 \%)$ in Down syndrome individuals than in the controls. Comparing the allelic and genotypic frequencies between the populations with Down syndrome and their parents with the controls using Pearson $\chi^{2}$ test and Fisher's exact test odds ratio, no statistically significant differences were found.

Conclusions. No statistically significant association was found between the polymorphisms of the APOE gene and Down syndrome. Sample size or ethnic influences may have affected these results.

\section{Contribución de los autores:}

Lucero Rengifo coordinó el proyecto, elaboró las historias clínicas de los pacientes con síndrome de Down y los estudios citogenéticos, hizo la revisión de la literatura científica y redactó el documento.

Duverney Gaviria practicó todos los estudios moleculares y colaboró con la elaboración del documento y el análisis de los resultados.

Herman Serrano revisó las bases de datos, elaboró las tablas e hizo los análisis estadísticos. 
More studies are necessary with other Colombian populations to determine possible associations in other genes related to Alzheimer's disease.

Key words: apolipoprotein E, Down syndrome, Alzheimer's disease, alleles, genotype, Colombia.

El síndrome de Down es la causa más frecuente de retardo mental de origen genético. Según los estudios de población, se presenta con una incidencia de 1 en 732 recién nacidos vivos (1), la cual se mantiene constante para los diferentes grupos raciales. De 90 a $95 \%$ presentan un cromosoma 21 extra y en el $96 \%$ de los casos este cromosoma extra es de origen materno debido a errores durante la ovogénesis (2). En estos casos, el $75 \%$ se atribuye a la falta de disyunción durante la meiosis I y, el $25 \%$ restante por errores en la meiosis II. La trisomía 21 de origen paterno se ha reportado en $5 \%$ de los casos por errores en la meiosis II $(3,4)$.

La edad materna avanzada ha sido bien documentada como factor de riesgo y se ha estimado que el riesgo para una mujer mayor de 35 años es de $1 / 385$, para una de 40 años es de $1 / 106$ y para una de 45 años es de $1 / 30$. No obstante, el $80 \%$ de los individuos con síndrome de Down nacen de mujeres jóvenes no mayores de 35 años (5). Además, la edad de las abuelas maternas en el momento de la concepción de la madre se ha relacionado como factor de riesgo (6).

La distribución de las variantes alélicas del gen de la apolipoproteína $\mathrm{E}(\mathrm{ApoE})$ se ha analizado en individuos con síndrome de Down de diferentes poblaciones y grupos étnicos. Se ha demostrado que en todos los individuos con este síndrome, existencambiosneuropatológicoscorrespondientes a la demencia de tipo Alzheimer, como son los depósitos del péptido $\beta$ amiloide extracelular en las placas neuríticas y los ovillos neurofibrilares intracelulares. La isoforma E4 de la apolipoproteína $\mathrm{E}$ se ha considerado como un factor de riesgo para padecer la enfermedad de Alzheimer. Aún se desconoce el mecanismo exacto por el cual ApoE influye en la presencia de Alzheimer; parece que promueve la formación y estabiliza la agregación de la proteína beta amiloide en las placas fibrilares que aparecen en la enfermedad de Alzheimer y el

\footnotetext{
Correspondencia:

Lucero Rengifo, Centro de Biología Molecular y Biotecnología, Facultad de Ciencias de la Salud, Universidad Tecnológica de Pereira, vereda La Julita, Pereira, Colombia

Telefax: (576) 321 5393, extensión 12

cenbiotep@utp.edu.co
}

Recibido: 30/08/11; aceptado:16/02/12 síndrome de Down. La proteína beta amiloide se considera el mayor componente proteico de estas placas y es un producto proteolítico del precursor de la proteína del péptido $\beta$ amiloide (7). El péptido $\beta$ amiloide empieza acumularse a partir de los 8 años y se incrementa con la edad. En individuos con síndrome de Down menores de 30 años, esta acumulación se manifiesta en forma de depósitos difusos que no están asociados con degeneración neurítica. Entre los 35 y 45 años, la acumulación del pépido $\beta$ amiloide se acelera y aparecen otros signos como la acumulación de los ovillos neurofibrilares y neuroinflamación (8).

Las manifestaciones neuropatológicas de la enfermedad de Alzheimer en el síndrome de Down se atribuyen a la triplicación y expresión incrementada del gen del precursor de la proteína $\beta$ amiloide localizado en el cromosoma 21 (9). Sin embargo, no todos los individuos con dicho síndrome desarrollan signos clínicos de enfermedad de Alzheimer. Diferentes estudios de población han demostrado que su prevalencia en individuos con síndrome de Down varía ampliamente (entre 6 y $75 \%)$ y se incrementa con la edad $(10,11)$. Esta variación se debe, posiblemente, a diferencias individuales de predisposición susceptibilidad a la formación de las placas fibrilares y del rango tan amplio de edad de manifestación de la demencia de tipo Alzheimer (9). Los síntomas de la enfermedad de Alzheimer en los individuos con síndrome de Down aparecen en edades más tempranas que en los individuos normales (10). La edad en la cual aparece la demencia varía entre los 38 y los 70 años, y la edad promedio se encuentra entre los 50 y los 55 años y muchos de ellos desarrollarían la enfermedad a los 80 años $(8,10)$.

Se han identificado varios genes candidatos a ser causantes de la susceptibilidad a desarrollar enfermedad de Alzheimer, tanto en la población general como en población con síndrome de Down. Estos estudios permitieron la identificación de 48 genes con valores LOD [logarithm (base 10) of odds], superiores a 2. Entre los genes que mostraron mayor relación estuvieron: $A p o E$ (gen de la apolipoproteína E), ApoC1 (gen de la apolipoproteína C1), TOMM40 (gen homólogo de la translocasa de la membrana mitocondrial externa 40 ), SORL1 (gen del receptor relacionado con sortilina), BACE 1 (gen de la enzima 1 que 
cliva el sitio $\beta$-péptido $\beta$ amiloide), RUNX1 (gen del factor 1 de transcripción relacionado con RUNT) y $A L D H 18 A 1$ (gen de la aldehído deshidrogenasa familia 18 , miembro A1) $(9,11)$.

El gen que codifica la apolipoproteína $\mathrm{E}(A p o E)$ tiene variantes alélicas conocidas como APOEع2, APOEع3 y APOE\&4 y una variante alélica rara de APOE\&3 conocida como APOE\&3r (12). Mediante estudios realizados en poblaciones de individuos con enfermedad de Alzheimer de diferentes orígenes geográficos y raza, incluyendo poblaciones colombianas, se ha demostrado que el alelo APOE\&4 es un factor de riesgo que influye en la edad de aparición de los síntomas (13-19). El alelo APOE\&4 se encuentra en el $50 \%$ de los individuos con enfermedad de Alzheimer de aparición tardía y la presencia de una copia de este alelo incrementa el riesgo de aparición tardía en tres veces y, dos copias, en 12 veces (18). Los individuos con aparición tardía que portan una o dos copias del alelo APOE\&4 desarrollan los síntomas de la enfermedad 10 a 20 años más temprano, comparados con los individuos que no llevan este alelo (15). El alelo APOEع2, menos común tanto en individuos con enfermedad de Alzheimer como en la población general, parece actuar como un factor protector para la aparición de la enfermedad $(20,21)$.

Se han encontrado frecuencias significativamente elevadas del alelo APOE\&4 y significativamente bajas del alelo APOEع2 en individuos con síndrome de Down que desarrollan enfermedad de Alzheimer antes de los 50 años de edad, comparados con individuos con síndrome que permanecen cognitivamente no afectados y con la población normal (22-24). En pruebas neuropsicológicas aplicadas a individuos adultos no dementes con síndrome de Down, se observó que la habilidad verbal estaba inversamente relacionada con la presencia de, al menos, un alelo APOE\&4. Los individuos con síndrome de Down no dementes con más edad (41 a 61 años) que tenían el alelo APOEع2, presentaban mejor comunicación verbal al compararlos con jóvenes (22 a 38 años) que portaban el alelo APOE\&4.

La relación entre la habilidad lingüística básica y el genotipo $A p o E$ indica que este factor genético influye en el desarrollo de la demencia y en la neuropatología de la enfermedad de Alzheimer en el síndrome de Down (25). Los cambios cognitivos relacionados con la edad en individuos con el síndrome no dementes, correspondieron con los cambios cognitivos tempranos de los individuos normales con enfermedad de Alzheimer (25). Además, el alelo APOE\&4 se ha asociado con mortalidad temprana y el alelo APOE\&2 con longevidad, tanto en la población general como en individuos con síndrome de Down $(24,26,27)$. Sin embargo, estudios similares de casos y controles, grupos familiares y por reportes de metaanálisis, no se ha encontrado asociación de los polimorfismos del gen $A p o E$ en individuos con síndrome de Down (28-31).

En los progenitores se ha encontrado aumento de la frecuencia del alelo APOE\&4 en madres jóvenes de individuos con síndrome de Down que desarrollan la enfermedad de Alzheimer (32). El alelo APOE\&3 se ha visto incrementado en madres y padres de individuos con síndrome de Down. Anello, et al. (31), encontraron tanto el genotipo $\varepsilon 3 / \varepsilon 3$ (odds ratio de 4,$\left.4 ; \mathrm{IC}_{95 \%} 3,4 \pm 5,7\right)$ como el alelo APOE\&3 elevados, en madres de individuos con síndrome de Down. La frecuencia del alelo APOE\&4 se ha encontrado aumentada en madres de individuos con síndrome de Down (32,33).

El objetivo de este estudio fue determinar la frecuencia de los alelos en los polimorfismos del gen $A p o E$ en pacientes con síndrome de Down y sus progenitores, y buscar una posible asociación entre los polimorfismos del gen $A p o E$ y dicho síndrome al comparar sus frecuencias con las de una población de individuos sanos.

\section{Materiales y métodos}

\section{Muestra}

Se hizo un muestreo por conveniencia en 134 individuos blanco-mestizos del eje cafetero con diagnóstico de síndrome de Down hecho mediante métodos clínicos y comprobado por el cariotipo con la técnica de bandas $\mathrm{G}$, en el Centro de Biología Molecular y Biotecnología de la Universidad Tecnológica de Pereira. Los pacientes provenían de tres centros de educación especial de los departamentos de Risaralda y Quindío, y de otros centros regionales de atención en salud. Las edades de los individuos con síndrome de Down oscilaba entre 2 días y 49 años (media de 10,51 años) y la distribución por sexo fue de 60 mujeres (45\%) y 74 hombres (55\%).

Los progenitores fueron 141 (54 padres y 87 madres). No fue posible estudiar todos los progenitores, ya que algunos no aceptaron participar en este estudio o estaban ausentes en el momento de la toma de muestra. 
Los análisis citogenéticos y moleculares se hicieron previo consentimiento informado de los progenitores o acudientes, el cual fue evaluado y aceptado por el Comité de Bioética de la Facultad de Ciencias de la Salud de la Universidad Tecnológica de Pereira.

La población control fue de 525 individuos del mismo grupo racial y origen geográfico que los pacientes con síndrome de Down y sus progenitores. Las edades de la población control, $223(42,5 \%)$ hombres y $302(57,5 \%)$ mujeres, oscilaba entre 3 y 50 años (media de 17,5 años); el dato se obtuvo de los registros de una muestra de población de individuos normales a los cuales se les hizo la genotipificación del gen $A p o E$ en un estudio anterior llevado a cabo en el Centro de Biología Molecular y Biotecnología de la Universidad Tecnológica de Pereira (34). Los datos de los controles se seleccionaron con una estrategia de generación de números aleatorios, utilizando la aplicación RAND del programa Excel.

\section{Genotipificación de ApoE}

Se tomaron $5 \mathrm{ml}$ de sangre de la vena del pliegue cubital en tubos Vacutainer ${ }^{\circledR}$ que contenían $\mathrm{K}_{2}$ EDTA como anticoagulante. Las muestras se almacenaron a $4{ }^{\circ} \mathrm{C}$ hasta el momento de la centrifugación para separar el plasma y el paquete de células blancas, en el cual se procedió a extraer el ADN utilizando el estuche comercial Puregene ${ }^{\circledR}$ (Gentra, Minneapolis, USA). Este estuche se basa en un método de extracción de proteínas no orgánico mediante el uso de sales que las precipita y permite purificar el ADN. El genotipo para ApoE se estableció mediante amplificación por PCR de un fragmento del gen $A p o E$ de 244 pares de bases y su posterior digestión con la enzima de restricción Hhal, como lo describen Hixson y Vernier (35), con algunas modificaciones (34).

Se usaron como iniciadores los oligonucleótidos F4 (5'-CAGAATTCGCCCCGGCCTGGTACAC-3') y F6 (5'-TAAGCTTGGCACGGCTGTCCAAGGA-3'). Cada reacción de amplificación contenía, además del amortiguador de amplificación, $1 \mu \mathrm{g}$ de ADN, 1 $\mathrm{pmol} / \mu \mathrm{l}$ de cada iniciador, dimetil-sulfóxido al $10 \%$ y 0,05 unidades de Taq polimerasa en un volumen final de $30 \mu \mathrm{l}$. El ADN se desnaturalizó a $95{ }^{\circ} \mathrm{C}$ durante un minuto y se programaron 30 ciclos de amplificación así: $95^{\circ} \mathrm{C}, 15$ segundos, $62{ }^{\circ} \mathrm{C}, 15$ segundos, y $72{ }^{\circ} \mathrm{C}, 15$ segundos.

Después de la amplificación por PCR se añadieron directamente a cada mezcla de reacción cinco unidades de la enzima de restricción Hhal, y se incubó a $37^{\circ} \mathrm{C}$ durante cuatro horas. Los fragmentos de restricción presentes en cada mezcla de reacción se separaron por electroforesis sin desnaturalización en geles de poliacrilamida al $15 \%(29: 1)$. Se utilizó como marcador de peso molecular el ADN de pBR322 digerido con Hae III. El gel se tiñó en una solución de bromuro de etidio $(1 \mu \mathrm{g} / \mathrm{ml})$ preparada en TBE $1 X$. Finalmente, los fragmentos de ADN se visualizaron en un transiluminador UV y se registraron los resultados utilizando fotografía digital. A partir del análisis del patrón de corte por la enzima de restricción, se estableció el genotipo (35).

\section{Análisis estadístico}

Se calcularon las frecuencias genotípicas y alélicas para las poblaciones de controles, casos y sus progenitores, con el paquete estadístico Statgraphics ${ }^{\mathrm{TM}}$, versión 5.1. Se hicieron análisis con el fin de determinar que las frecuencias alélicas y genotípicas en las poblaciones se encontraran en equilibrio de Hardy-Weinberg, utilizando pruebas de $\chi^{2}$ para la bondad de ajuste. Mediante el uso de pruebas $\chi^{2}$ de Pearson para tablas de contingencia, se hicieron comparaciones entre las poblaciones. Estos análisis se llevaron a cabo tanto en la población general como al separarla por sexo. Finalmente, se evaluó el odds ratio (OR) para el alelo y genotipo más frecuente en la población de individuos con síndrome de Down.

\section{Resultados}

Se analizaron por conteo directo las frecuencias alélicas y genotípicas de los polimorfismos en el gen $A p o E$ para las poblaciones con síndrome de Down, sus progenitores y controles, como se describen en el cuadro 1. Algunos de los resultados de las genotipificaciones por PCR-RFLP se muestran en la figura 1. Las poblaciones de casos y sus progenitores se encontraron en equilibrio de Hardy-Weinberg $(p=0,97$, madres $p=0,96$ y padres $p=0,96)$. En la población de controles las frecuencias genotípicas para $A p o E$ se encontraron en desequilibrio $(p=0,0008)$ a expensas de un mayor número de individuos homocigóticos $\varepsilon 2 / \varepsilon 2$.

En la población con síndrome de Down se encontró que el alelo más frecuente fue el APOE\&3 (85\%) $y$ el menos frecuente fue el alelo APOE\&2 $(4,1$ $\%)$ (cuadro 1). La frecuencia del alelo APOE\&4 se observó más elevada que en el resto de las poblaciones analizadas (11\%). El genotipo más frecuente fue $\varepsilon 3 / \varepsilon 3$ (72\%), seguido del genotipo $\varepsilon 3 / \varepsilon 4$ (18\%). Los otros genotipos mostraron 
Cuadro 1. Frecuencias genotípicas y alélicas de los polimorfismos en el gen ApoE en las poblaciones estudiadas

\begin{tabular}{|c|c|c|c|c|c|c|c|c|c|c|c|c|c|c|c|c|}
\hline \multirow[b]{3}{*}{ Casos } & \multicolumn{10}{|c|}{ Frecuencias genotípicas (\%) } & \multicolumn{6}{|c|}{ Frecuencias alélicas (\%) } \\
\hline & \multirow{2}{*}{$\begin{array}{c}\mathbf{n} \\
134\end{array}$} & \multirow{2}{*}{$\begin{array}{l}2 / 2 \\
0\end{array}$} & $2 / 3$ & \multicolumn{2}{|c|}{$2 / 4$} & $3 / 3$ & \multicolumn{2}{|c|}{$3 / 4$} & \multicolumn{2}{|c|}{$4 / 4$} & \multirow{2}{*}{$\begin{array}{c}\mathbf{n} \\
268\end{array}$} & \multirow{2}{*}{\multicolumn{2}{|c|}{$\begin{array}{c}2 \\
11 \quad(4,1)\end{array}$}} & \multirow{2}{*}{$\begin{array}{c}3 \\
227(85)\end{array}$} & \multirow{2}{*}{\multicolumn{2}{|c|}{$\begin{array}{ll} & 4 \\
30 \quad(11)\end{array}$}} \\
\hline & & & $9(7,0)$ & 2 & $(1,5)$ & $97(72)$ & 24 & (18) & 2 & $(1,5)$ & & & & & & \\
\hline Controles & 525 & $8(1,5)$ & $45(8,6)$ & 10 & $(1,9)$ & 381 (72) & 74 & (14) & 7 & $(1,3)$ & 1.050 & 71 & $(7,0)$ & 881 (84) & 98 & $(9,0)$ \\
\hline Madres & 87 & 0 & $6(7,0)$ & 0 & & $68(78)$ & 12 & (14) & 1 & $(1,0)$ & 174 & 6 & $(3,0)$ & $154(90)$ & 14 & $(8,0)$ \\
\hline Mujeres controles & 302 & $5(1,6)$ & $24(8,0)$ & 4 & $(1,3)$ & $222(73)$ & 42 & (14) & 5 & $(2,0)$ & 604 & 38 & $(6,0)$ & $510(84)$ & 56 & $(9,3)$ \\
\hline Padres & 54 & 0 & $4(7,4)$ & 0 & & 41 (76) & 9 & (17) & 0 & & 108 & 4 & $(4,0)$ & $95(88)$ & 9 & $(8,0)$ \\
\hline Hombres controles & 223 & $3(1,3)$ & $21 \quad(9.0)$ & 6 & $(3,0)$ & $159(70)$ & 32 & (14) & 2 & $(0,9)$ & 446 & 33 & $(7,0)$ & 371 (83) & 42 & $(9,0)$ \\
\hline
\end{tabular}

frecuencias muy bajas y el genotipo $\varepsilon 2 / \varepsilon 2$ estuvo ausente. En la población control el alelo más frecuente fue el APOEع3 (84\%), el genotipo más frecuente fue $\varepsilon 3 / \varepsilon 3$ (72\%), seguido de $\varepsilon 3 / \varepsilon 4$ (14\%) y $\varepsilon 2 / \varepsilon 3(8,6 \%)$. En esta población se encontró el genotipo $\varepsilon 2 / \varepsilon 2$ con una frecuencia baja, de 1,5\%.

Al hacer las comparaciones entre las poblaciones de casos y controles utilizando la $\chi^{2}$ de Pearson (cuadro 2) y el cálculo de los OR con el uso de la prueba exacta de Fisher, tanto para el alelo APOE\&4 (OR=1,22; IC $\mathrm{IC}_{95 \%}$ 1,887-0,859; $\left.\mathrm{p}=0,35\right)$ como para el alelo APOE 22 no se encontraron diferencias significativas $\left(\mathrm{OR}=0,59 ; \mathrm{IC}_{95 \%}, 1,130\right.$ 0,$347 ; p=0,120$ ).

En la población de progenitores tanto en madres como en padres, el alelo más frecuente fue APOE\&3 (90\% y $88 \%$, respectivamente) y el alelo APOE\&2 fue el menos frecuente ( $3 \%$ en madres y $4 \%$ en padres). Los genotipos más frecuentes en los progenitores fueron el $\varepsilon 3 / \varepsilon 3$ (78 \% en madres y $76 \%$ en padres) seguido del genotipo $\varepsilon 3 / \varepsilon 4$ (14\% en madres y $17 \%$ en padres). En ambos progenitores estuvieron ausentes los genotipos $\varepsilon 2 / \varepsilon 2$, $\varepsilon 2 / \varepsilon 4$ y en padres estuvo ausente el genotipo $\varepsilon 4 / \varepsilon 4$.

Para el análisis la población control se separó por sexo encontrándose que tanto en las mujeres como en los hombres el alelo más frecuente fue el APOE\&3 ( $84 \%$ y $83 \%$, respectivamente) y el menos frecuente fue el alelo APOE\&2 (6\% en mujeres y $7 \%$ en hombres). Los genotipos más frecuentes tanto en mujeres como en hombres fueron $\varepsilon 3 / \varepsilon 3$ (73\% y $70 \%$ ) y $\varepsilon 3 / \varepsilon 4$ con la misma frecuencia del $14 \%$. Cuando se hicieron las comparaciones entre las poblaciones de madres con mujeres de la población control y padres con hombres de la población control, no se encontraron diferencias estadísticamente significativas al evaluar genotipos y alelos (cuadro 2). El alelo APOE\&4 se encontró con la misma frecuencia en ambos progenitores (8 $\%)$, y con frecuencias muy similares, en mujeres y hombres de la población control.
Como los alelos APOع3, APO 44 y el genotipo $\varepsilon 3 / \varepsilon 3$ se encontraron incrementados significativamente en los progenitores de individuos con síndrome de Down, se hizo una comparación entre las poblaciones de madres de hijos con síndrome de Down y mujeres de la población control para los alelos APO\&3 (OR=1,42; $I_{95 \%}$ 2,375-0,931; $\mathrm{p}=0,223), \mathrm{APO} \varepsilon 4$ (OR=0,86; IC 95\% 1,578-0,519,

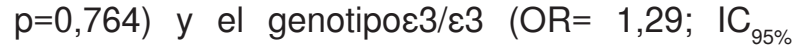
$2,279-0,810 ; p=0,406)$ y no hubo diferencias significativas. En padres de hijos con síndrome de Down y hombres de la población control no se encontraron diferencias significativas para el alelo APO\&3 (OR=1,27; $\left.I C_{95 \%} 2,252-0,723 ; p=0,244\right)$, el alelo APOع4 (OR=0,920; IC $\left.{ }_{95 \%} 1,96-0,50 ; p=1,0\right)$ y el genotipo $\varepsilon 3 / \varepsilon 3\left(\mathrm{OR}=1,27 ; \mathrm{IC}_{95 \%} 2,252-0,723\right.$; $p=0,612$ ).

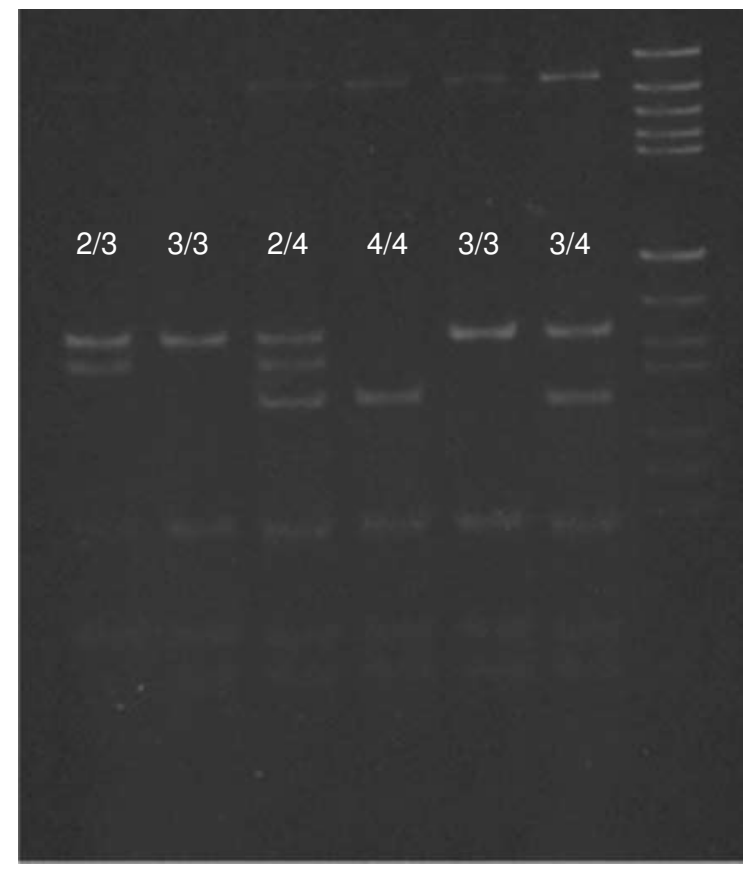

Figura 1. Electroforesis en gel de poliacrilamida al $12 \%$, teñido con bromuro de etidio. Carriles 1 al 6: polimorfismos en el gen de APOE; carril 7: marcador de peso molecular pBR322 digerido con Hae III. 
Debido a que la edad materna es un factor de riesgo bien estudiado para engendrar descendientes con síndrome de Down, se dividió la población en madres jóvenes ( $\leq 35$ años) y madres mayores (>35 años) en el momento de la concepción, para averiguar si existía alguna relación de propensión con los polimorfismos del gen ApoE (cuadro 3). Sin embargo, la comparación de las frecuencias de los alelos y genotipos entre estos dos grupos no fue diferente (datos no mostrados).

\section{Discusión}

Varios estudios de población se han centrado en la correlación entre los polimorfismos del gen $A p o E$ y la demencia de tipo Alzheimer en individuos adultos con síndrome de Down, con resultados contradictorios (13-17,23,29-31,36,37). Deb, et al. (36), reportaron un metaanálisis de 1.132 individuos con síndrome de Down, 316 individuos con síndrome de Down y demencia de tipo Alzheimer y 816 individuos con síndrome de Down sin demencia. En este metaanálisis encontraron un exceso estadísticamente significativo del alelo APOE\&4 en individuos adultos con síndrome de Down y con demencia de tipo Alzheimer. Sin embargo, no observaron una disminución significativa del alelo APOEع2 en esta misma población. En los metaanálisis descritos por Prasher, et al. (29), y van Goo,l et al. (37), no encontraron asociación del alelo APOE\&4 con la enfermedad de Alzheimer en individuos adultos con síndrome de Down.

Anello, et al. (30), encontraron una frecuencia baja del alelo APOE\&4 y una elevada del alelo APOE\&3 en pacientes con síndrome de Down, comparados con controles, y una frecuencia del genotipo $\varepsilon 3 /$ ¿4 baja en pacientes con síndrome de Down comparados con controles. Estos resultados, según los autores, podrían deberse a las diferencias en las edades de los pacientes, a las características de la población control que no fue apareada por edad y sexo, y al impacto del origen racial de la población estudiada, incluyendo características genéticas y factores ambientales desconocidos.

Lambert, et al. (31), no encontraron asociación entre los polimorfismos del gen ApoE y el síndrome de Down en una población europea de 41 individuos con este síndrome entre 45 y 52 años con y sin demencia de tipo Alzheimer, y una población de 35 controles apareados por edad y sexo. Resultados similares se observaron en este estudio, en el cual no se encontraron diferencias estadísticamente significativas en las frecuencias del alelo APOE\&4 en individuos con síndrome de Down al compararlos con una población control.

La frecuencia del alelo APOE\&4 en individuos con síndrome de Down con demencia de tipo Alzheimer

Cuadro 2. Comparación de las frecuencias genotípicas entre las poblaciones de casos y controles utilizando una prueba de $\chi^{2}$ de Pearson (valor de $p$ )

\begin{tabular}{|c|c|c|c|c|c|c|c|c|c|c|c|}
\hline & $\mathbf{n}$ & $2 / 2$ & $2 / 3$ & $2 / 4$ & $3 / 3$ & $3 / 4$ & $4 / 4$ & $\mathbf{n}$ & 2 & 3 & 4 \\
\hline Casos & 134 & & & & & & & & & & \\
\hline & & 0,15 & 0,48 & 0,75 & 0,96 & 0,26 & 0,88 & 268 & 0,11 & 0,23 & 0,36 \\
\hline Controles & 525 & & & & & & & 1.050 & & & \\
\hline Madres & 87 & & & & & & & 174 & & & \\
\hline & & 0,22 & 0,75 & 0,28 & 0,38 & 0,97 & 0,73 & & 0,15 & 0,18 & 0,61 \\
\hline Mujeres controles & 302 & & & & & & & 604 & & & \\
\hline Padres & 54 & & & & & & & 108 & & & \\
\hline Hombres controles & 223 & 0,39 & 0,64 & 0,22 & 0,49 & 0,67 & 0,48 & 446 & 0,16 & 0,22 & 0,72 \\
\hline
\end{tabular}

Cuadro 3. Grupos etarios de las madres al momento del parto y genotipificación del gen ApoE

\begin{tabular}{|c|c|c|c|c|c|c|c|c|c|c|}
\hline \multirow[b]{2}{*}{$\begin{array}{l}\text { Grupos etarios } \\
\text { (años) }\end{array}$} & \multirow[b]{2}{*}{$\mathrm{n}=87$} & \multicolumn{6}{|c|}{ Genotipos } & \multicolumn{3}{|c|}{ Alelos } \\
\hline & & $2 / 2$ & $2 / 3$ & $2 / 4$ & $3 / 3$ & $3 / 4$ & $4 / 4$ & 2 & 3 & 4 \\
\hline 14 a 20 & 12 & 0 & 1 & 0 & 11 & 0 & 0 & 1 & 23 & 0 \\
\hline 21 a 25 & 9 & 0 & 1 & 0 & 6 & 2 & 0 & 1 & 15 & 2 \\
\hline 26 a 30 & 11 & 0 & 0 & 0 & 9 & 2 & 0 & 0 & 20 & 2 \\
\hline 31 a 35 & 15 & 0 & 1 & 0 & 11 & 3 & 0 & 1 & 26 & 3 \\
\hline 36 a 40 & 26 & 0 & 3 & 0 & 21 & 2 & 0 & 3 & 47 & 4 \\
\hline 41 a 45 & 11 & 0 & 0 & 0 & 8 & 2 & 1 & 0 & 18 & 4 \\
\hline 46 a 50 & 3 & 0 & 2 & 0 & 1 & 0 & 0 & 2 & 4 & 0 \\
\hline
\end{tabular}


y sin ella, oscila entre 3 y $33 \%$ (32). En este estudio la frecuencia del alelo el APOE\&4 fue de 11 $\%$ en los individuos con síndrome de Down y de 9 $\%$ en la población control. Este incremento en los individuos con síndrome de Down es a expensas del genotipo $\varepsilon 3 / \varepsilon 4$ que se encuentra más elevado en esta población (18\%) que en la población control (14\%), aunque no significativamente. En los controles y los progenitores el alelo APOE\&4 presenta frecuencias muy similares, al igual que los genotipos $\varepsilon 3 / \varepsilon 4$ y $\varepsilon 4 / \varepsilon 4$.

La frecuencia del alelo APOEع2 fue baja, prácticamente en todas las poblaciones analizadas. Sin embargo, en la población control se encontraba más elevada $(7,0 \%)$ aunque no de manera significativa, en comparación con los individuos con síndrome de Down (4,1\%). Este alelo se encuentra aún más bajo en la población de madres (3,0 \%). El genotipo $\varepsilon 2 / \varepsilon 2$ está ausente en las poblaciones con síndrome de Down y sus progenitores, y el genotipo $\varepsilon 2 / \varepsilon 4$, en las madres. En el metaanálisis reportado por Deb et al. (36), la frecuencia del alelo APOEع2 oscila entre 3 y $11,8 \%$ en los individuos con demencia de tipo Alzheimer y entre 2,4 y 50 $\%$ en los individuos con síndrome de Down no dementes. En un estudio más reciente de 100 individuos adultos con síndrome de Down con demencia de tipo Alzheimer y sin ella y que fueron separados por sexo, encontraron una frecuencia de $4 \%$ en el alelo APOE\&2 en individuos con síndrome de Down y enfermedad de Alzheimer, mientras que en los no dementes la frecuencia fue de $13 \%$. Las mujeres presentaron un riesgo de 1,77 veces mayor que los hombres de desarrollar la demencia de tipo Alzheimer (28).

El alelo APOE\&3 presentó frecuencias muy similares en las poblaciones evaluadas, al igual que los genotipos $\varepsilon 3 / \varepsilon 3, \varepsilon 3 / \varepsilon 4, \varepsilon 2 / \varepsilon 3$. Este alelo es el más frecuentemente encontrado en todos los estudios de población. Se ha reportado elevación significativa del genotipo $\varepsilon 3 / \varepsilon 3$ y los alelos APOE\&3 y APOE\&4, en madres de individuos con síndrome de Down $(30,32,33)$. En este estudio no se encontraron diferencias estadísticamente significativas en las frecuencias alélicas y genotípicas del gen $A p o E$ en los progenitores de los individuos con síndrome de Down, comparados con la población normal.

En estudios previos de casos y controles en poblaciones colombianas con enfermedad de Alzheimer, encontramos frecuencias muy parecidas de los alelos APOE\&2, APOE\&3, tanto en la población afectada como en controles. Sin embargo, hay diferencias relacionadas con la frecuencia del alelo APOE\&4 que es mayor en los individuos con enfermedad de Alzheimer. Como en nuestra población de individuos con síndrome de Down y progenitores, el genotipo $\varepsilon 2 / \varepsilon 2$ está ausente tanto en individuos con enfermedad de Alzheimer como en la población control. Se observa elevación de la frecuencia del genotipo $\varepsilon 3 / \varepsilon 4$ en individuos con enfermedad de Alzheimer y baja en los controles $(16,19)$.

En 10 comunidades amerindias, 6 poblaciones afrodescendientes y una población mestiza colombianas, se encontraron los tres alelos APOE\&2, APOE\&3 y APOE\&4. En la mayoría de las comunidades amerindias estuvo ausente el alelo APOEع2, mientras que el alelo APOE\&4 presentó la frecuencia más elevada. En las poblaciones afrodescendientes, el alelo APOEع2 se presentó con una frecuencia elevada del $20 \%$ y el alelo APOE\&4 osciló entre 14 y $26 \%$. En la población mestiza las frecuencias de los alelos APOE\&2 y APOE\&4 fueron de $10 \%$ y $11,5 \%$, respectivamente (38), similares a las frecuencias reportadas en este estudio. No obstante, las frecuencias alélicas son diferentes a las reportadas para las poblaciones afrodescendientes.

En este estudio no se encontró asociación entre los polimorfismos del gen $A p o E$ y el síndrome de Down. Es probable que por las características de la muestra, como el tamaño y el hecho de que fuera un muestreo por conveniencia, no pueda considerarse representativa de toda la población con síndrome de Down en esta región. Otros factores importantes que se deben considerar y que podrían influir en estos resultados, son la variabilidad étnica de la población colombiana y las características genéticas.

Es necesario llevar a cabo más estudios en otras poblaciones colombianas con síndrome de Down en los que se analicen los polimorfismos del gen $A p o E$ que ayuden a sustentar estos resultados. Es igualmente importante seguir evaluando en esta población la relación del síndrome de Down y la enfermedad de Alzheimer con los polimorfismos en los genes APOC1 y TOMM40 asociados con enfermedad de Alzheimer al igual que el alelo APOE\&3r. Por ser esta una población joven (edad promedio 10,51 años), se debe hacer un estudio prospectivo, tanto en la población con síndrome de Down como en la de sus progenitores, relacionado con deterioro cognitivo, desarrollo de enfermedad de Alzheimer y mortalidad. 


\section{Agradecimientos}

Los autores agradecen la colaboración que prestaron directivos y docentes de las instituciones CINDES, IQEE y RENACER, a los padres y familiares de los pacientes.

\section{Conflicto de intereses}

Los autores manifiestan que no existe conflicto de intereses relacionados con este trabajo.

\section{Financiación}

Este estudio fue financiado por la Vicerrectoría de Investigaciones de la Universidad Tecnológica de Pereira.

\section{Referencias}

1. Sherman SL, Allen MG, Bean LH, Freeman SB. Epidemiology of Down syndrome. Ment Retard Dev D R. 2007;13:221-7.

2. Allen EG, Freeman SB, Druschel C, Hobbs CA, O'Leary LA, Romitti PA, et al. Maternal age and risk for trisomy 21 assessed by the origin of chromosome nondisjuntion: A report from the Atlanta and National Down Syndrome Projects. Hum Genet. 2009;125:41-2.

3. Antonarakis SE, Petersen MB, Mclnnis MG, Adelsberger PA, Schinzel AA, Binkert F, et al. The meiotic stage of nondisjunction in trisomy 21: Determination by using DNA polymorphisms. Am J Hum Genet. 1992;50:544-50.

4. Sherman SL, Petersen MB, Freeman SB, Hersey J, Pettay D, Taft M, et al. Non-disjunction of chromosome 21 in maternal meiosis I: Evidence for a maternal agedependent mechanism involving reduced recombination. Hum Mol Genet. 1994;3:1529-35.

5. Petersen MB, Mikkelsen M. Nondisjuntion in trisomy 21 : Origin and mechanism. Cytogenet Cell Genet. 2000;91:199203.

6. Malini SS, Ramachandra NB. Influence of advanced age of maternal grandmothers on Down syndrome. BMC Med Genet. 2006;7:1-5.

7. Dupuy AM, Mas E, Ritchie K, Descomps B, Badiou S, Cristol JP, et al. The relationship between apolipoprotein E4 and lipid metabolism is impaired in Alzheimer's disease. Gerontology. 2001;47:213-8.

8. Tyrrell J, Cosgrave M, McCarron M, McPherson J, Calvert J, Kelly A, et al. Dementia in people with Down's syndrome. Int J Geriatr Psychiat. 2001;16:1168-74.

9. Patel A, Rees SD, Kelly MA, Bain SC, Barnett AH, Thalitaya $\mathrm{D}$, et al. Association of variants within APOE, SORL1, RUNX1, BACE1 and ALDH18A1 with dementia in Alzheimer's disease in subjects with Down syndrome. Neurosci Lett. 2011;487:144-8.

10. Visser FE, Aldenkamp AP, van Huffelen AC, Kuilman M, Overweg J, van Wijk J. Prospective study of the prevalence of Alzheimer type dementia in institutionalized individuals with Down syndrome. Am J Ment Retard. 1997;101:400-12.

11. Chu SH, Roeder K, Ferrell RE, Devlin B, DeMichele-Sweet MA, Kamboh Ml et al. TOMM40 poly-T repeat lengths, age of onset and psychosis risk in Alzheimer disease. Neurobiol Aging. 2011;32:2328:e1-9.

12. Seripa D, Matera MG, Daniele A, Bizzarro A, Rinaldi MC, Gravina L, et al. The missing ApoE allele. Ann Hum Genet. 2007;71:496-500.

13. Sando SB, Melquist S, Cannon A, Hutton ML, Sletvold O, Saltvedt I, et al. APOE epsilon 4 lowers age at onset and is a high risk factor for Alzheimer's disease; a case control study from central Norway. BMC Neurol. 2008;8:1-7.

14. Khachaturian AS, Corcoran CD, Mayer LS, Zandi PP, Breitner JC. Apolipoprotein E epsilon4 count affects age at onset of Alzheimer disease, but not lifetime susceptibility: The Cache County Study. Arch Gen Psychiat. 2004;5:518-24.

15. Corder A, Saunders W, Strittmatter D, Schmechel P, Gaskell G, Small G, et al. Gene dose of apolipoprotein E type 4 allele and the risk of Alzheimer's disease in late onset families. Science. 1993;261:921-3.

16. Jacquier M, Villareal E, Torres $\mathbf{O}$, Serrano ML, Cruts $\mathbf{M}$, Montañes P, et al. APOE epsilon4 and Alzheimer's disease: Positive association in a Colombian clinical series and review of the Latin-American studies. Arq Neuropsiquiatr. $2001 ; 59: 11-7$

17. Huang Y. Apolipoprotein E and Alzheimer disease. Neurology. 2006;66(Suppl.1):S79-85.

18. Verghese PB, Castellano JM, Holtzman DM. Apoliprotein $\mathrm{E}$ in Alzheimer's disease and other neurological disorders. Lancet Neurol. 2011;10:241-52.

19. Arboleda GH, Yunis JJ, Pardo R, Gómez CM, Hedmont D, Arango G, et al. Apolipoprotein $\mathrm{E}$ genotyping in a sample of Colombian patients with Alzheimer's disease. Neurosci Lett. 2001;135-8.

20. Corder E, Saunders A, Risch M, Strittmatter W, Schmechel D, Gaskell P, et al. Protective effect of apolipoprotein $\mathrm{E}$ type 2 allele for late onset Alzheimer disease. Nat Genet. 1994;7:180-4.

21. Trachtenberg AJ, Filippini N, Cheeseman J, Duff EP, Neville MJ, Ebmeier KP, et al. The effects of APOE on brain activity do no simply reflect the risk of Alzheimer's disease. Neurobiol Aging. 2012;618:e1-13.

22. Coppus AM, Evenhuis HM, Verberne GJ, Visser FE, Vásquez AA, Sayed-Tabatabaei $F$, et al. The impact of apolipoprotein $\mathrm{E}$ on dementia in persons with Down's syndrome. Neurobiol Aging. 2008;29:828-35.

23. Tyrell j, Cosgrave M, Hawi Z, McPherson J, O’Brien C, McCalvert $\mathrm{J}$, et al. A protective effect of apolipoprotein $\mathrm{E}$ e2 allele on dementia in Down's syndrome. Biol Psychiatry. 1998;43:397-400.

24. Prasher VP, Sajith SG, Rees SD, Patel A, Tewari S, Schupf $\mathbf{N}$, et al. Significant effect of APOE epsilon 4 genotype on the risk of dementia in Alzheimer's disease and mortality in persons with Down syndrome. Int $\mathrm{J}$ Geriatr Psychiatry. 2008;11:1134-40.

25. Alexander GE, Saunders AM, Szczepanik J, Strassburger TL, Pietrini P, Danni A, et al. Relation of age and apolipoprotein $\mathrm{E}$ to cognitive function in Down syndrome adults. Neuroreport. 1997;8:1835-40.

26. Zigman WB, Jenkins EC, Tycko B, Schupf N, Silverman W. Mortality is associated with apolipoprotein E epsilon4 in 
non demented adults with Down syndrome. Neurosci Lett. 2005;390:93-7.

27. Schupf N, Sergievsky GH. Genetic and host factors for dementia in Down's syndrome. $\mathrm{Br} J$ Psychiatry 2002;180:405-10.

28. Lai F, Kammann E, Rebeck GW, Anderson A, Chen Y, Nixon RA. APOE genotype and gender effects on Alzheimer disease in 100 adults with Down syndrome. Neurology. 1999;53:331-6.

29. Prasher VP Chowdhury T, Rowe B, Bain S. ApoE genotype and Alzheimer's disease in adults with Down syndrome: Meta-analysis. Am J Ment Retard. 1997;102:103-10.

30. Anello G, Guéant J, Romano C, Barone C, Pettinato R, Pillot T, et al. Allele e4 of apolipoprotein E gene is less frequent in Down syndrome patients of the Sicilian population and has no influence on the grade of mental retardation. Neurosci Lett. 2001;306:129-1.

31. Lambert JC, Tur JP, Duperi MJ, Delacourte A, Frigard B, Chatier-Harlim MC. Analysis of the APOE alleles impact in Down's syndrome. Neurosci Lett. 1996; 220:57-60.

32. Schupf N, Kapell D, Lee J, Ottman R, Mayeus R. Increased risk of Alzheimer's disease in mothers of adults with Down's syndrome. Lancet 1994;344: 353-6.
33. Avramopoulos D, Mikkelsen M, Vassilopoulos D, Grigoriadou M, Petersen M. Apolipoprotein E allele distribution in parents of Down's syndrome children. Lancet. 1996;347:862-5.

34. Rodríguez J, Cediel V. Genotipificación de apolipoproteína E en la población de Risaralda. Rev Med Risaralda. 1999;5:2-7.

35. Hixon J, Vernier V. Restriction isotyping of human apolipoporotien $\mathrm{E}$ by gene amplification and cleavage with Hhal. J Lipid Res. 1990;31:545-8.

36. Deb S, Braganza J, Norton N, Williams H, Kehoe PG, Williams $\mathrm{J}$, et al. ApoE e4 influences the manifestation of Alzheimer's disease in adults with Down's syndrome. Br J Psychiatry. 2000;176:468-72.

37. van Gool WA, Evenhuis HM, van Duijn CM. A case-control study of apolipoprotein E genotypes in Alzheimer's disease associated with Down's syndrome. Dutch Study Group on Down's Syndrome and Ageing. Ann Neurol. 1995;38:22530.

38. Jaramillo-Correa JP, Keyeux G, Ruiz-García M, Rodas C, Bernal J. Population genetic análisis of the genes APOE, APOB ( $3^{\prime}$ VNTR) and ACE in some black and Amerindian communities from Colombia. Hum Hered. 2001;52:14-33. 